\title{
e-Migrinter
}

$18 \mid 2019$

L'ethnographie en migration(s)

\section{Didier Fassin (2018) La vie. Mode d'emploi critique}

\author{
Lydie Déaux
}

\section{OpenEdition}

Journals

Édition électronique

URL : https://journals.openedition.org/e-migrinter/1538

DOI : 10.4000/e-migrinter.1538

ISSN : 1961-9685

Éditeur

UMR 7301 - Migrinter

Référence électronique

Lydie Déaux, « Didier Fassin (2018) La vie. Mode d'emploi critique », e-Migrinter [En ligne], 18| 2019, mis en ligne le 11 septembre 2019, consulté le 20 mai 2021. URL : http://journals.openedition.org/emigrinter/1538; DOI : https://doi.org/10.4000/e-migrinter.1538

Ce document a été généré automatiquement le 20 mai 2021.

Tous droits réservés 


\title{
Didier Fassin (2018) La vie. Mode d'emploi critique
}

\author{
Lydie Déaux
}

\section{RÉFÉRENCE}

Fassin, Didier (2018) La vie. Mode d'emploi critique, Condé-sur-Noireau, Seuil, 192 p. (La couleur des idées).

Les « Adorno Lectures » qui se tiennent à l'Institut für Sozialforschung de l'Université de Goethe de Francfort en 2016 rendent hommage à Theodor Adorno, philosophe, sociologue et musicologue. Le cycle de conférence de Didier Fassin sur la «vie » est retranscrit dans cet ouvrage. Symptomatique de sa carrière professionnelle ${ }^{1}$, la vie est aussi l'objet récurent et central des études ethnographiques qu'il mène depuis maintenant deux décennies.

2 Plus que de comprendre ce qu'est une vie "bonne ", "juste », ou encore le sens de celle-ci, Didier Fassin amène le lecteur à se questionner sur la valeur des vies et les inégalités entre elles. Tout au long d'un plaidoyer qui allie philosophie, anthropologie et sociologie, il nous donne les clés de compréhension des hiérarchies morales qui fondent les inégalités de vie.

Pour mieux situer le lecteur dans le cadre conceptuel et théorique de l'ouvrage, Didier Fassin, dans un préambule assez délayé, revient sur les définitions que donnent certains sociologues, anthropologues, littéraires et philosophes de «la vie » (Adorno (1951), Locke (1689), Canguilhem (1952), Arendt (1958), Proust (1927), Beckett (1951), Bourdieu (1986), Agamben (1995), etc.). Il découpe la suite de son ouvrage en trois chapitres distincts qui abordent les formes, les éthiques et les politiques de vie. Si ces trois thèmes semblent interroger des problématiques opposées, «l'inégalité des vies » est le fil rouge habilement tracé par Fassin qui conduit le lecteur à penser leur articulation. 
Didier Fassin respecte la même construction dans les trois chapitres: l'introduction reprend les principaux concepts philosophiques qui se rattachent au thème abordé, la partie empirique permet d'illustrer et d'ancrer son propos dans la réalité sociale observée, la conclusion présente les limites, apports et approfondissements possibles de sa réflexion.

5 À l'aune de ce premier chapitre, le lecteur décèle l'intention de Didier Fassin de démontrer les expériences précaires et situations juridiques que peuvent partager les migrants ou "nomades forcés", comme il convient de les nommer selon lui. Son développement démontre néanmoins les situations multiples et diverses de ces personnes migrantes, donc des différentes formes de vie qui résident dans une expérience qui semble, aux premiers abords, commune et consensuelle. Cette analyse critique renvoie les démocraties actuelles dans les confins et limites qu'elles présentent en termes de gestion des populations, et par extension, des vies.

6 Certains scientifiques se sont attachés à hiérarchiser les différentes formes de vie organiques selon des caractéristiques externes et objectives (comme les mammifères). À l'inverse, les sciences sociales justifient l'existences de plusieurs formes de vie selon des différences internes et subjectives. Cette tension renvoie à l'ancien débat anthropologique qui oppose la nature à la culture comme justification de l'existence de différentes formes de vie et à leur hiérarchisation.

7 Plutôt que de penser ces théories en opposition radicale, Didier Fassin propose de les percevoir comme en "tension productive ", éclairage nouveau dans la conception des formes de vie. Autrement dit, il convient de penser les formes de vies dans leur diversité plutôt que dans leur divergence.

8 À l'aide d'exemples sur les syriens réfugiés à Calais et les zambiennes en situation irrégulière à Johannesburg, Didier Fassin s'attarde sur la raison de leur présence plus que sur les points communs de ces situations migratoires d'apparence individuelle et particulière.

9 Les apports théoriques de Wittgenstein $(1953,1977)$, Canguilhem $(1952,1968)$ et Agamben $(2011,2014)$ ainsi qu'un triple rapport dialectique détaillé permettent l'articulation des concepts mobilisés pour éclairer ce questionnement.

10 Le premier rapport, ou "couple antagonique», est celui entre l'universel et le particulier. Tous les migrants éprouvent l'instabilité de leur statut juridique, de leur position sociale. Cependant, leur trajectoire et socialisation, propre à chacun, rendra le partage et l'écoute par autrui de cette expérience migratoire parfois impossible.

11 La tension entre le biologique et le biographique met en lumière l'universalité et la centralité de la « survie » chez les personnes migrantes, sans pour autant omettre que la réponse à leurs besoins primaires passe par des interactions et rapports sociaux avec certains acteurs spécifiques (policiers, membres des ONG, etc.).

12 Les stratégies d'adaptations, l'accommodement que font certains migrants face aux obstacles dressés par le droit, notamment vis-à-vis du statut légal, montrent bien le décalage qui existe entre la loi et la pratique, en tant que tension productive.

13 Les formes de vie selon Didier Fassin nourrissent l'appréhension de certains concepts qui composent le champ des migrations internationales, tel que le «parcours migratoire » et « l'autonomie». 
14 Le deuxième chapitre expose plusieurs éthiques de vie plus ou moins acceptées par l'imaginaire collectif de nos sociétés. Aux débats disciplinaires sur les formes de vie s'ajoute ainsi celui du « sens » de la vie, question universelle et intemporelle. À travers le postulat que plus la vie est pensée et valorisée de manière abstraite, plus les inégalités de traitement de la valeur des vies seront visibles, Didier Fassin nous présente deux terrains ethnographiques différents à titre illustratif et explicatif.

Il soulève la tendance des politiques de gestion des migrations en France à octroyer plus facilement une régularisation du titre de séjour aux étrangers se réclamant de la raison humanitaire (Fassin, 2010), à l'inverse du statut de réfugié politique. La valeur sociale et politique de la vie s'efface derrière la valeur biologique et naturelle, ce qui crée une véritable «biolégitimité » indiscutable quand il s'agit de gestion des populations migrantes. En Afrique du Sud, l'accès à des soins et droits sociaux est facilité pour les personnes atteintes du SIDA, à l'inverse des minorités victimes d'inégalités sociales - notamment de santé - dans une période post-apartheid. A travers le terme de "citoyenneté biologique ", on constate un processus de "réduction de la vie à son expression physique » (Fassin, 2018 : 90), où le caractère biologique de la vie prime de nouveau sur le social et le politique. L'auteur rappelle la distinction entre vie biologique (zô̂) et vie sociale (bios) en transposant les apports d'Agamben et de Foucault à ses recherches.

Pour éclairer les perceptions divergentes portées sur les éthiques de vies, il compare le geste humanitaire qui consiste à sauver la vie d'autrui et le geste de certains militants qui sacrifient leurs vies pour celle des autres (comme les palestiniens sur les terres occupées). Si ces deux exemples ont en commun l'instrumentalisation de la vie en tant que l'investissement éthique ultime, d'un côté «le fait de garder en vie » et de l'autre " de mourir pour une cause », le premier exemple fait beaucoup plus consensus que le deuxième (Fassin, 2018 : 104).

17 En opposant le concept de «biopouvoir» (ou «biopolitique ») de Michel Foucault avec celui de «politiques de vies », Didier Fassin distingue, dans ce troisième chapitre, les logiques de gestion des vies des populations avec les processus de traitements et significations différenciés des vies. Accorder des valeurs différentes aux vies selon un traitement inégal entre directement en opposition avec la conceptualisation éthique de celle-ci comme bien suprême de nos sociétés. Cette tension est issue de l'inclusion de la monétarisation pour compenser et réparer les vies humaines. De choquantes, nécessaires à insuffisantes, les compensations financières se sont institutionnalisées au cours de l'Histoire, creusant toujours plus d'écart dans la différence de valeur accordée aux vies. Il développe plusieurs exemples probants comme celui la dot, des compensations juridiques pour réparation aux victimes ou encore des assurances vies.

18 Deux terrains d'enquêtes éclairent la compréhension plus générale de ce processus de justification de la différenciation des vies : un immigré héroïnomane sidéen qui, en perdant son titre de séjour, ne peut se soigner et meurt jeune ; un ouvrier sud-africain qui réside sur son lieu de travail, attrape le virus du sida en fréquentant des prostituées, perd ainsi son travail et finit par vivre dans une cabane isolée, seul et sans interaction sociale.

19 Sont ainsi mises en avant l'inégalité par l'exclusion (la vie de l'immigré résidant en France n'a aucune valeur pour la société et peut même représenter un coût) et l'inégalité par l'exploitation (atteint du virus du SIDA, l'ouvrier sud-africain perd sa force de travail, sa vie étant réduite à cette seule valeur). L'idée que la dévalorisation de 
ces vies permet la justification des traitements inégaux qui leurs sont imposés (Fassin, 2018 : 144-145) est un signal d'alarme fort lancé aux lecteurs.

Didier Fassin conclut ce chapitre sur la double dimension de l'espérance de vie, à prendre au sens propre comme au figuré ; la première permet la mesure de la quantité de vie (nombre moyen d'années d'existence), la seconde sa qualité (l'espérance comme forme d'attente de nos vies qui dépend de nos interrelations avec autrui).

21 Plutôt qu'une lecture misérabiliste des vies des exilés ou "nomades forcés " qui penserait leurs vies comme vulnérables et précaires, Didier Fassin propose une compréhension des vies contextualisées dans des rapports sociaux de domination qui affirment le caractère inégal de celles-ci. Cet ouvrage est finalement une critique de la non-reconnaissance de ce processus de traitement différencié des vies mis en place par les politiques et ancré dans l'imaginaire collectif de nos sociétés. L'idéalisation de la vie biologique empêche la considération des vies biographiques. Plus qu'un dialogue transdisciplinaire - parfois dense, dont l'abondance tend quelque peu à perdre le lecteur dans les références mentionnées - Didier Fassin s'est confronté à une problématique trop souvent occultée. Quelque que soit l'objet de recherche en migrations internationales ou la discipline dans laquelle on s'inscrit, cet ouvrage est un excellent préambule de réflexions à quiconque souhaite s'interroger sur les vies et leurs inégalités de traitement.

\section{NOTES}

1. Après une carrière en médecine, Didier Fassin s'est progressivement tourné vers l'anthropologie, la sociologie et la philosophie.

\section{AUTEURS}

\section{LYDIE DÉAUX}

doctorante en sociologie, Laboratoire Migrinter (UMR 7301) CNRS / Université de Poitiers lydie.deaux@univ-poitiers.fr 